\title{
Mechanism for Damage to DNA by Low-Energy Electrons ${ }^{\dagger}$
}

\author{
Robyn Barrios, Piotr Skurski, and Jack Simons* \\ Chemistry Department and Henry Eyring Center for Theoretical Chemistry, University of Utah, \\ Salt Lake City, Utah 84112
}

Received: October 17, 2001; In Final Form: December 18, 2001

\begin{abstract}
We have carried out ab initio electronic structure calculations on a portion of DNA, the results of which provide support for a mechanism that produces single-strand breaks (SSBs) with low-energy electrons. This mechanism involves attaching a low-energy electron (ca. $1 \mathrm{eV}$ ) to a $\pi^{*}$ orbital of a DNA base to form a shape-resonance state. This $\pi^{*}$ anion then undergoes a sugar-phosphate $\mathrm{C}-\mathrm{O}$ bond rupture over a small barrier to produce SSBs. In addition to supporting the efficacy of such a mechanism, our results suggest that solvation plays a crucial role in the rate of SSB formation when such very short-lived shape resonances are involved. In particular, they suggest that either the $\pi^{*}$ anion must be rendered electronically stable by solvation or its detachment lifetime must be several orders of magnitude longer in the solvated species than in the nonsolvated species.
\end{abstract}

\section{Introduction}

It has been suggested ${ }^{1}$ that ionizing radiation impinging on living cells produces secondary electrons with kinetic energies in the $1-20 \mathrm{eV}$ range and that it is these low-energy electrons that cause many of the lethal DNA lesions ${ }^{2}$ (i.e., single- and double-strand breaks, SSBs and DSBs, respectively). Secondary electrons in the above energy range are created in numbers ${ }^{3}$ $\left(\sim 5 \times 10^{4}\right.$ per $\mathrm{MeV}$ of deposited energy) that make them the most abundant species created by ionizing $\left(\mathrm{x}^{-}, \gamma^{-}\right.$, or $\left.\beta^{-}\right)$ radiation. The yields of SSBs and DSBs caused by such electrons have been observed ${ }^{2}$ to be $8.2 \times 10^{-4}$ and $2 \times 10^{-4}$ strand breaks per electron, respectively. These values are $10-$ 100 times the yields induced by even higher-energy (10-25 $\mathrm{eV}$ ) photons. It also was noted that the electrons need not possess energy in excess of DNA's ionization threshold (7.5-10 eV) to induce these strand breaks.

In recent experiments from the Sanche group, ${ }^{2}$ solid samples of clean DNA containing its structural water ${ }^{4}$ were irradiated with beams of low-energy electrons for a specific duration at a beam current density of $2.2 \times 10^{12}$ electrons $\mathrm{s}^{-1} \mathrm{~cm}^{-2}$ and a well-defined kinetic energy $E$. After irradiation, the DNA samples were tested for single- and double-strand breaks. The energy dependence of the observed SSB and DSB yields showed

1. that SSBs and DSBs occur even for rather low-energy electrons $(E \approx 5-7 \mathrm{eV}$, which is below the $7.5 \mathrm{eV}$ ionization threshold of DNA);

2. that the SSB and DSB yields depend on the electron beam energy $E$ in a manner that suggested attachment of the electrons in some resonance process (i.e., there were peaks in the plots of yield vs $E$ ); and

3. that no significant SSBs for electrons with energies in the $1 \mathrm{eV}$ range (i.e., in the range studied in the present paper) occurred.

In other recent work, it has been suggested ${ }^{5}$ that core-excited (two-electron, one-hole) resonance states were most likely involved in this $(>5 \mathrm{eV})$ energy range. The Sanche group

\footnotetext{
$\dagger$ Part of the special issue "John C. Tully Festschrift".

* Corresponding author. E-mail: simons@chemistry.chem.utah.edu.
}

concluded that the DNA SSBs are initiated by resonant electron attachment to some DNA components (base, deoxyribose, phosphate, or hydration water), followed by bond dissociation. They also suggest that DSBs are caused by subsequent reactions of the products of SSBs with other DNA components.

In the present study, we examined the kind of mechanism suggested in the Sanche work in further detail using ab initio electronic structure methods that include solvation effects. However, we focused on processes occurring at even lower electron energies where attachment into the lowest $\pi^{*}$ orbitals of conjugated fragments ${ }^{6}$ of DNA is most plausible. ${ }^{7}$ That is, we examined the $\pi^{*}$ shape-resonance state rather than the higher-energy core-excited resonances of ref 2 . Our efforts are directed toward probing the potential energy landscape of such anions formed via electron attachment to $\pi^{*}$ orbitals both in the absence and in the presence of aqueous solvation. More precisely, we explored the nature of the energy surface along a coordinate that leads to the kind of SSBs observed ${ }^{8}$ in radiationinduced lesions in DNA. Our findings suggest that

1. SSBs are very unlikely to occur at rates competitive with the rates of electron detachment from the metastable 1-eV shaperesonance anion in the absence of water solvent but

2. SSBs can indeed occur at rates consistent with observed yields and via the proposed resonant capture mechanism for $1-\mathrm{eV}$ electrons if the DNA is stabilized by water solvation.

These data thus suggest that the evidence provided for coreexcited resonances being involved in strand breaks likely extends to even lower-energy electrons that produce shape resonances but only if the DNA is strongly solvated.

\section{Methods}

To examine the entire DNA molecule using the type of ab initio electronic structure tools needed for this study is currently prohibitive. Therefore, we had to select a portion of the full DNA molecule that would be representative both of the electron attachment and the $\mathrm{C}-\mathrm{O}$ bond rupture events that we wish to examine. In this our first study of the possibilities that present themselves, we chose to excise a portion of the DNA containing 


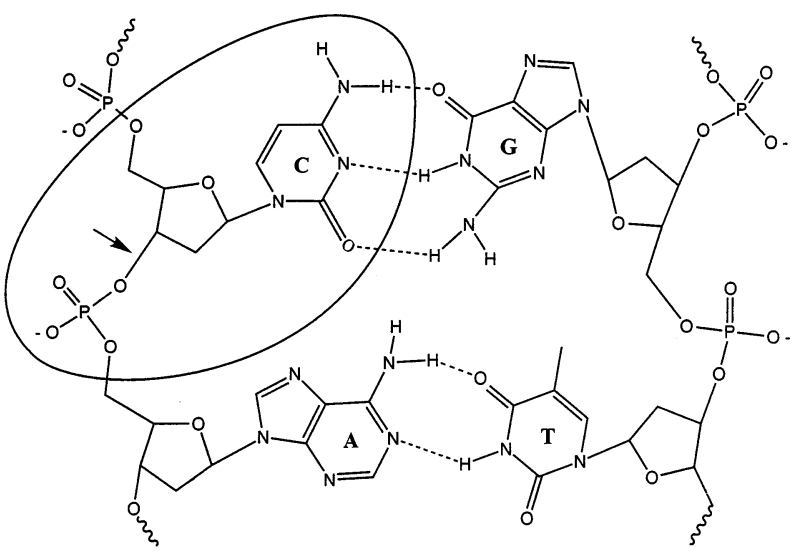

Figure 1. Fragment excised from DNA containing cytosine base, sugar, and phosphate groups. The $\mathrm{C}-\mathrm{O}$ bond cleaved in SSB formation is marked with an arrow.

1. a cytosine base that contains the delocalized orbital system to whose $\pi^{*}$ orbital an electron would be attached and

2. a sugar moiety characteristic of all such fragments of DNA, which connects the cytosine to

3. a phosphate group attached to the sugar by the $\mathrm{C}-\mathrm{O}$ bond that is ruptured in SSBs. In Figure 1, the fragment we excised from DNA to study ${ }^{9}$ in this research effort is shown, and the $\mathrm{C}-\mathrm{O}$ bond broken in SSBs is labeled with an arrow.

Having chosen the representative fragment, our strategy was to proceed as follows:

1. We "terminated" the $-\mathrm{C}-\mathrm{O} \bullet$ and $-\mathrm{OPO}_{3}{ }^{-1}$ radical centers (formed when we cut bonds within the DNA) of our fragment by adding $\mathrm{H}$ atoms; we also protonated the $-\mathrm{OPO}_{3}{ }^{-1}$ anion site to neutralize it. ${ }^{10}$ The former was done to eliminate the radical centers generated by our artificial excising of the fragment from intact DNA. Such centers, if not so terminated, would provide artificial electron attachment sites that would obscure the $\pi^{*}$ binding site we wish to emphasize.

2. We carried out a series of ab initio electronic structure calculations on the neutral and anion formed by adding an electron to the $\pi^{*}$ LUMO. For the neutral,

a. the sugar-phosphate $\mathrm{C}-\mathrm{O}$ bond length $R$ was stretched, in steps of $0.05 \AA$, from $1.40 \AA$, which is near its equilibrium length, to large distances $(2.20 \AA)$ where the $\mathrm{C}-\mathrm{O}$ bond is broken and the SSB has occurred.

b. At each value of $R$, the other internal coordinates of the neutral DNA fragment were varied to minimize the electronic energy (computed at the restricted self-consistent field (RHF) level for this closed-shell species).

3 . For the $\pi^{*}$ anion, we repeated steps a and $b$ above but used the unrestricted Hartree-Fock (UHF) approximation for this open-shell system.

In this manner, we generated a "slice" through the potential energy surface of the anion fragment that connects the $\pi^{*}$ anion to the bond-ruptured SSB, as well as an analogous slice for the neutral.

4. We carried out an independent set of such calculations on the $\pi^{*}$ anion and the neutral in the presence of solvating water molecules and with no solvent present (so we could determine whether solvation was crucial to the proposed mechanism's efficacy). In these calculations, we observed that the internal geometries of the solvated and unsolvated species were quite similar.

As noted above, the electronic energy $E(R)$ at each value of $R$ was computed by optimizing the remaining internal coordinates using the SCF method with a $6-31+\mathrm{G}^{*}$ atomic orbital basis set, ${ }^{11}$ the RHF and UHF methods being used on the neutral and anion, respectively. Because the $\pi^{*}$ anion is not an electronically stable species but is metastable with respect to electron loss, we had to take additional measures. In particular, we selected the above rather modest basis not only because it is small enough to render our calculations feasible but also because it produced a $\pi^{*}$ LUMO with an energy near $1 \mathrm{eV}$. This energy is near where the experiments determined there to be a shape-resonance state. ${ }^{6}$ Thus, this basis allowed us to simulate the behavior of a $\pi^{*}$ state formed by attaching $1-\mathrm{eV}$ electrons. Another attractive feature of this basis set is that the $\pi^{*}$ anion was electronically stable at the Koopmans' theorem level even though it is unstable by ca. $1 \mathrm{eV}$ at the SCF level. This fact allowed us to identify efficiently the cytosine $\pi^{*}$ orbital that should be occupied to compute the energy of the metastable anion.

One may wonder why we did not choose to use a correlated treatment such as a density functional theory (DFT) treatment. Certainly, such calculations would not have been significantly more computer-intensive. However, we deemed this approach not to be optimal because of the well-known difficulties in interpreting the DFT orbital energies. That is, because DFT orbital energies do not offer zeroth-order approximations to electron binding energies, we knew it would be difficult to identify properly the desired 1-eV shape-resonance state that we wanted to study. For these reasons, we limited our calculations to the SCF level.

To describe the effect of surrounding water molecules on the electronic energy and geometry of our model DNA fragment, we employed the polarized continuum (PCM) solvation model ${ }^{12}$ within a self-consistent reaction field treatment, and we performed all calculations using the Gaussian 98 program. ${ }^{13}$ Because of the size of our model system, it would be computationally prohibitive to include in our ab initio calculations the large number of water molecules needed to solvate the DNA fully. Moreover, even to include explicitly the ca. 2.5 water molecules per base thought to be present in ref 2's experiments would have been difficult to simulate. Hence, we were forced to use as simple and cost-effective a solvation model as was reasonable.

Because of the large size of the DNA fragment, the nature of the metastable $\pi^{*}$ state, and our desire to include solvation, we have been forced to use theoretical methods (small basis sets, neglect of electron correlation, approximate treatment of solvation) that cannot be expected to provide quantitatively accurate predictions. As such, our approach has been designed to produce results that may suggest whether the proposed electron attachment to form a $\pi^{*}$ shape resonance followed by $\mathrm{C}-\mathrm{O}$ bond rupture could be a mechanism for SSBs in DNA. Our results also provide us an opportunity to follow the attached electron's charge distribution as SSBs take place and to monitor the energy profile during this process, thus gaining important mechanistic detail.

\section{Results}

A. Energy Profiles. In Figure 2 are shown plots of the electronic energies of the neutral and $\pi^{*}$ anion shape-resonance species both with full aqueous (PCM) solvation and without solvent present. The neutral-fragment plots show that it is energetically quite endothermic (by 35 and $27 \mathrm{kcal} \mathrm{mol}^{-1}$, without and with solvation, respectively) to rupture the $\mathrm{C}-\mathrm{O}$ bond that is broken to produce SSBs. Thus, it is unlikely that SSBs can occur at $298 \mathrm{~K}$ (where the experiments of ref 2 took place) or even at significantly elevated temperature if the 


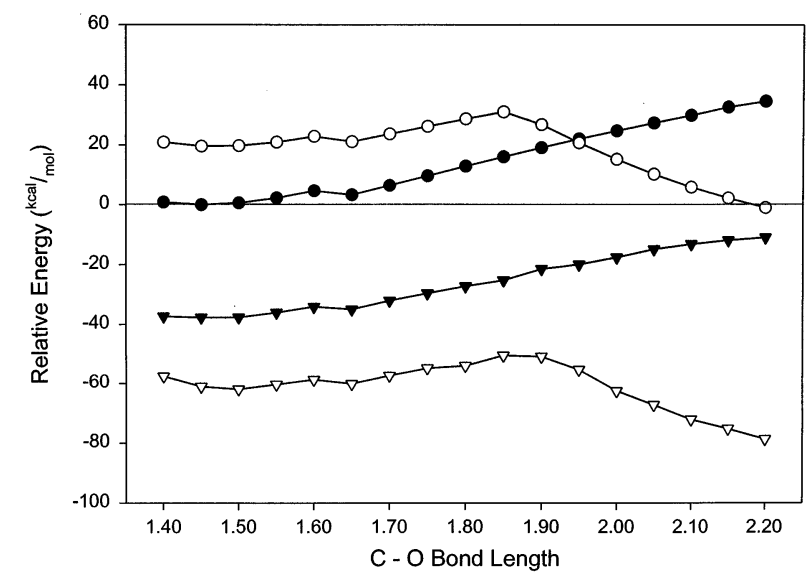

Figure 2. Plots of SCF energies $\left(\mathrm{kcal} \mathrm{mol}^{-1}\right)$ of unsolvated neutral $(\bullet)$ and anionic $(O)$ species as well as the fully solvated neutral $(\boldsymbol{\nabla})$ and anion $(\nabla)$ vs the $\mathrm{C}-\mathrm{O}$ bond length $(\AA)$.
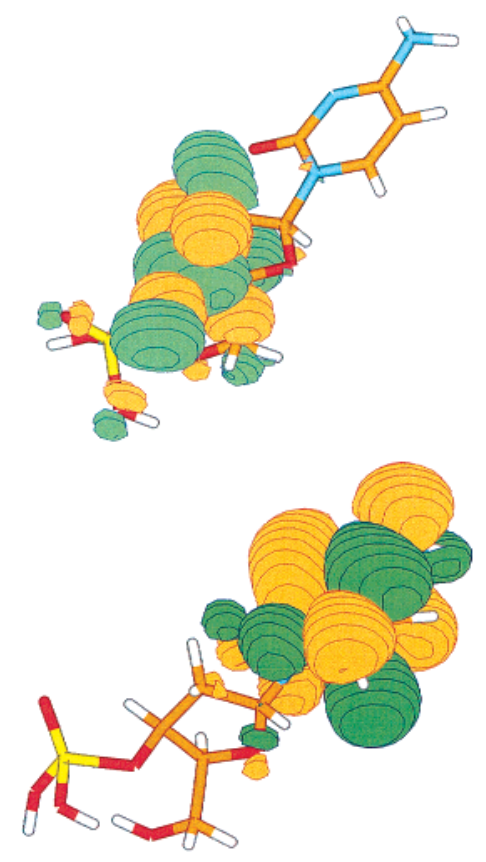

Figure 3. Singly occupied molecular orbital (SOMO) of the solvated anion computed at the equilibrium $R_{\mathrm{CO}}$ distance (bottom) showing the $\pi^{*}$ orbital and when the $\mathrm{C}-\mathrm{O}$ bond has ruptured (top).

fragment remains neutral (i.e., does not attach an electron), whether strong solvation is present or not.

The $\pi^{*}$ anion energy profile in the absence of water solvent suggests that $\mathrm{C}-\mathrm{O}$ bond rupture requires surmounting a $13 \mathrm{kcal}$ $\mathrm{mol}^{-1}$ barrier but that the fragmentation process is exothermic ${ }^{14}$ by $21 \mathrm{kcal} \mathrm{mol}^{-1}$. The exothermicity results from the large electron affinity of the "phosphate" group $-\mathrm{O}-\mathrm{PO}_{3} \mathrm{H}_{2}$ generated by bond rupture and the negative electron affinity of the cytosine group's $\pi^{*}$ shape resonance to which the electron initially attaches.

In Figure 3, we show the singly occupied molecular orbital ${ }^{15}$ (SOMO) of our anion fragment (in the presence of solvation; the unsolvated case shows similar behavior) near the equilibrium value of $R$, where the electron clearly is in the cytosine $\pi^{*}$ orbital, and at large $R$, where the electron resides between the phosphate and sugar groups.

These plots clearly show that the attached electron can indeed migrate "smoothly" from the $\pi^{*}$ orbital to the phosphate, but the rate of such charge migration will depend on the rate at which the barrier on the surface is surmounted. In examining this SOMO at various $R$ values, we found that the character remained much like that shown in the top of Figure 3 (i.e., $\pi^{*}$ like) for all $R$ values between 1.40 and $1.85 \AA$. However, for $R$ greater than or equal to $1.90 \AA$ (actually, beyond $R=1.864$ $\AA$ ), the SOMO was essentially identical to that shown at the bottom of Figure 3. That is, the shift of electron density from the cytosine $\pi^{*}$ region onto the sugar and phosphate region occurs over a very narrow range of $R$ values near $1.864 \AA$.

The energy profile of the solvated anion shows a slightly reduced barrier of $12 \mathrm{kcal} \mathrm{mol}^{-1}$ and an exothermicity ${ }^{14}$ of 17 $\mathrm{kcal} \mathrm{mol}^{-1}$. However, the primary difference between the nonsolvated and fully solvated data is that the $\pi^{*}$ anion lies below the neutral in the latter, whereas in the former, the anion is a metastable resonance state. This difference is crucial because, for the metastable anion, SSB must occur at a rate that is competitive with electron detachment; for the fully solvated anion, SSB need not compete with autodetachment of the $\pi^{*}$ state to be observed.

B. Rates of SSB Versus Rates of Detachment. Let us now consider whether it is plausible that a nascent $\pi^{*}$ anion shape resonance at ca. $1 \mathrm{eV}$, without water solvent, could undergo $\mathrm{C}-\mathrm{O}$ bond rupture to form SSB fragments within the expected lifetimes $\left(10^{-13}\right.$ to $\left.10^{-14} \mathrm{~s}\right)$ of such metastable shape-resonance $\pi^{*}$ states. Recall that the yield of SSBs per attached electron seems to be in the $10^{-3}$ to $10^{-4}$ range (at least for the resonances observed in ref 2). Therefore, the rate of SSB formation should be 3 to 4 orders of magnitude less than the rate of electron detachment. Thus, we would require the rate of SSB formation to be $\left(10^{-3}\right.$ to $\left.10^{-4}\right) \times\left(10^{13}\right.$ to $\left.10^{14}\right) \mathrm{s}^{-1}=10^{9}$ to $10^{11} \mathrm{~s}^{-1}$ to produce the proper branching ratio between detachment and SSB formation.

To estimate the rate of SSB formation, we consider the vibrations of the $\mathrm{C}-\mathrm{O}$ bond that must rupture. This bond's vibrational motion occurs near $1000 \mathrm{~cm}^{-1}$ in infrared spectra, so such bonds vibrate at a rate of ca. $3 \times 10^{13} \mathrm{~s}^{-1}$. The probability $P$ that this $\mathrm{C}-\mathrm{O}$ bond stretches, through thermal activation at $298 \mathrm{~K}$, enough to surmount the $13 \mathrm{kcal} \mathrm{mol}^{-1}$ barrier shown in Figure 2 is $P=\exp (-13(503) / 298)=3 \times$ $10^{-10}$. Hence, the average rate of SSB formation would be expected to be ca. $3 \times 10^{13}\left(3 \times 10^{-10}\right) \approx 10^{4} \mathrm{~s}^{-1}$, so if the $\pi^{*}$ anion's detachment rate is as specified above, the rate of SSB formation would be too slow by 5 or more orders of magnitude to be consistent with the observed SSB yields. Thus, it seems unlikely that SSBs could be induced by electron attachment to the cytosine base's $\pi^{*}$ orbital forming a shape resonance near $1 \mathrm{eV}$ if the system is not solvated. This idea is, in fact, consistent with the observations of ref 2, where no SSBs were observed for $1-\mathrm{eV}$ electrons.

However, if the $\pi^{*}$ anion is electronically stable, as it is when fully solvated where the barrier ${ }^{16}$ is $12 \mathrm{kcal} \mathrm{mol}^{-1}$, the probability of reaching the barrier would be $P=2 \times 10^{-9}$, and the SSB rate would be $6 \times 10^{4} \mathrm{~s}^{-1}$. In this case, competition with autodetachment is not an issue, and SSBs can occur at the above rate. Hence, if the rate of electron loss from the $\pi^{*}$ anion (not due to autodetachment but to processes such as radical scavenging or chemical decay) were in the $10^{8}-10^{9} \mathrm{~s}^{-1}$ range and the SSB rate were $6 \times 10^{4} \mathrm{~s}^{-1}$, as computed above, the observed branching ratio for SSBs could occur. These ideas suggest that the experiments of ref 2 be repeated, if possible, with samples containing substantial amounts of water solvent to see whether 1-eV electrons can induce SSBs.

In conclusion, it is indeed plausible that SSBs can occur at rates consistent with what is known from experiments by a 
mechanism involving $\pi^{*}$ shape resonances. However, this can happen only if the $\pi^{*}$ anion is rendered stable or metastable with a lifetime $\left(10^{-8}-10^{-9} \mathrm{~s}\right)$ with respect to any decay process that is considerably longer than that in the unsolvated case.

\section{Summary}

Our ab initio simulations of SSBs induced by attaching an electron to the $\pi^{*}$ orbital of cytosine to form a shape-resonance state suggest that a mechanism analogous to that suggested earlier ${ }^{2}$ for SSBs in DNA induced by low-energy electrons may be operative. The generalization offered in our work applies to the lower-energy (ca. $1 \mathrm{eV}$ ) shape-resonance case rather than to the higher-energy $(>5 \mathrm{eV})$ core-excited resonances examined in ref 2. Specifically, our results allow us to postulate the following:

1. Solvation is necessary for very low-energy electrons (i.e., in the few-eV range) to induce SSBs via $\pi^{*}$ shape resonances. The unsolvated fragment examined here has too large a barrier to surmount to produce SSBs at a rate competive with that of autodetachment, which, we suggest, is why the relatively "dry" DNA samples used in ref 2 did not display significant SSBs for $1-\mathrm{eV}$ electrons.

2. Solvation of the $\pi^{*}$ anion and of the SSB's products likely, depending on the degree of solvation, renders the anion either electronically stable or longer-lived than that in the nonsolvated case. Thus, solvation is key to allowing the $\pi^{*}$ state to produce SSBs at any significant rate.

3. The attached electron evolves, over a very narrow range of geometries, from occupying a cytosine $\pi^{*}$ orbital in the initially formed anion to occupying an orbital between the sugar and phosphate groups in the SSB products.

The first and second points raised above suggest that SSB formation (induced by low-energy electrons in $\pi^{*}$ shape resonances) could be reduced by mutating the base components of DNA in a way that shortens their detachment lifetimes (e.g., by adding electron-donating $\mathrm{H}_{3} \mathrm{C}-$ groups). Conversely, any mutation (e.g., adding halogen substituents) that renders the $\pi^{*}$ state electronically stable or very long-lived can be expected to increase SSB damage rates.

Finally, we wish to reemphasize that, although suggestive of the likely mechanism of SSB in DNA caused by low-energy electrons in $\pi^{*}$ states, the findings of this initial effort are by no means definitive. It is our plan to extend these studies by

1. examining other $\pi^{*}$ fragments excised from DNA (e.g., fragments containing thymine, guanine, and adenine);

2 . adjusting the orbital exponents of the more diffuse $\pi$ basis functions on the base moiety to adjust the energy of the $\pi^{*}$ orbital to thus consider electron attachment at various energies $E$ (to see how the barrier, and thus the SSB rate, depends on $E$ within such usually very broad shape resonances); and

3. using ab initio molecular dynamics (rather than simply scanning the energy surface along a single coordinate) to follow the fragmentation of the $\pi^{*}$ anion to determine if bonds other than the $\mathrm{C}-\mathrm{O}$ bond shown in Figure 1 are likely to rupture.

Acknowledgment. This work was supported by NSF grant \#9982420 to J.S., by the University of Utah's ACCESS program for young female scholars, and by a grant from the Pfizer Corporation to R.B. The computer time provided by the Center for High Performance Computing at the University of Utah is also gratefully acknowledged.

\section{References and Notes}

(1) Radiation Damage in DNA: Structure/Function Relationships at Early Times; Fuciarelli, A. F., Zimbrick, J. D., Eds.; Battelle: Columbus,
OH, 1995. von Sonntag, C. The Chemical Basis for Radiation Biology; Taylor and Francis: London, 1987.

(2) Boudaiffa, B.; Cloutier, P.; Hunting, D.; Huels, M. A.; Sanche, L. Science (Washington, D.C.) 2000, 287(5458), 1658.

(3) Pimblott, S. M.; LaVerne, J. A. Radiation Damage in DNA: Structure/Function Relationships at Early Times; Fuciarelli, A. F., Zimbrick, J. D., Eds.; Battelle: Columbus, OH, 1995; p 3.

(4) The samples used in ref 2 are thought to contain ca. 2.5 water molecules per base pair.

(5) Abdoul-Carime, H.; Cloutier, P.; Sanche, L. Radiat. Res. 2001, 155, 625. In this article, the authors suggest that core-excited resonances are likely involved both because of the energy range and because such resonaces are known to have longer lifetimes than shape resonances (a longer lifetime allows dissociation to compete with autodetachment).

(6) Attachment of low-energy electrons to DNA bases that has been studied using electron transmission spectroscopy is reported in Aflatooni, K.; Gallup, G. A.; Burrow, P. D. J. Phys. Chem. A 1998, 102, 6205.

(7) Among all the components of DNA, including its water solvent, it is the $\pi$ orbitals of the bases that produce the lowest-energy unoccupied molecular orbitals (LUMOs), the $\pi^{*}$ orbitals.

(8) Stubbe, J.; Kozarich, J. W. Chem. Rev. 1987, 87, 1107. Giloni, L.; Takeshita, M.; Johnson, F.; Iden, C.; Grollman, A. P. J. Biol. Chem. 1984, $236,8608$.

(9) We should note that, in subsequent studies, we plan to carry out similar analyses of different fragments. For example, fragments containing thymine, guanine, or adenine will be considered.

(10) The protonation was carried out to produce a more realistic electrostatic potential for the electron that we attached to the cytosine group to form the $\pi^{*}$ anion that we desired to study. It is likely that, in the native DNA, this phosphate anion site is solvated and screened by counterions to an extent that it no longer appears to be a negative-ion site to the electron occupying cytosine's $\pi^{*}$ orbital. For this reason, we thought it wise to so protonate this site.

(11) Ditchfield, J. R.; Hehre, W. J.; Pople, J. A. J. Chem. Phys. 1971, 54, 724. Hehre, W. J.; Ditchfield, R.; Pople, J. A. J. Chem. Phys. 1972, 56, 2257.

(12) In implementing the PCM calculation, we employed a dielectric constant (EPS $=78$ ) that is representative of water solvation and the default (sphere around each solute heavy atom) definition of the solvation cavity's shape and size. Although this likely is too strong a solvation to replicate the environment of the DNA tested in ref 2, we wanted to compare the behavior of our fragment at two extremes: nonsolvated and fully solvated as in aqueous solution. References to the PCM model include Miertus, S.; Scrocco, E.; Tomasi, J. Chem. Phys. 1992, 55, 117. Miertus, S.; Tomasi, J. Chem. Phys. 1982, 65, 239. Cossi, M.; Barone, V.; Cammi, R.; Tomasi, J. Chem. Phys. Lett. 1996, 255, 327.

(13) Frisch, M. J.; Trucks, G. W.; Schlegel, H. B.; Scuseria, G. E.; Robb, M. A.; Cheeseman, J. R.; Zakrzewski, V. G.; Montgomery, J. A., Jr.; Stratmann, R. E.; Burant, J. C.; Dapprich, S.; Millam, J. M.; Daniels, A. D.; Kudin, K. N.; Strain, M. C.; Farkas, O.; Tomasi, J.; Barone, V.; Cossi, M.; Cammi, R.; Mennucci, B.; Pomelli, C.; Adamo, C.; Clifford, S.; Ochterski, J.; Petersson, G. A.; Ayala, P. Y.; Cui, Q.; Morokuma, K.; Malick, D. K.; Rabuck, A. D.; Raghavachari, K.; Foresman, J. B.; Cioslowski, J.; Ortiz, J. V.; Stefanov, B. B.; Liu, G.; Liashenko, A.; Piskorz, P.; Komaromi, I.; Gomperts, R.; Martin, R. L.; Fox, D. J.; Keith, T.; Al-Laham, M. A.; Peng, C. Y.; Nanayakkara, A.; Gonzalez, C.; Challacombe, M.; Gill, P. M. W.; Johnson, B. G.; Chen, W.; Wong, M. W.; Andres, J. L.; Head-Gordon, M.; Replogle, E. S.; Pople, J. A. Gaussian 98, revision A.7; Gaussian, Inc.: Pittsburgh, PA, 1998.

(14) This exothermicity is the energy released when one moves R from its equilibrium value to $2.2 \AA$, where the $\mathrm{C}-\mathrm{O}$ bond is essentially broken. However, even at $2.2 \AA$, there remains significant electrostatic and hydrogen bonding attraction between the phosphate fragment and the remaining group. For the solvated species, only $17 \mathrm{kcal} \mathrm{mol}^{-1}$ is required to break the $\mathrm{C}-\mathrm{O}$ bond, but subsequent removal of the phosphate ion asymptotically is 22 kcal mol ${ }^{-1}$ more exothermic. This extra exothermicity arises because asymptotically both the phosphate ion and the remaining cytosine plus sugar radical can be stabilized by solvation.

(15) These plots were obtained using the MOLDEN program: Schaftenaar. G.; Noordik, J. H. J. Comput.-Aided Mol. Des. 2000, 14, 123.

(16) The absolute accuracy of our calculated energy profile is quite limited, and the barrier computed in the manner used here certainly results in an over-estimate of the true barrier. That is, we compute $E$ by varying one coordinate (the $\mathrm{C}-\mathrm{O}$ distance) and optimizing all other distances until we reach a local maximum. The true reaction path search involves choosing a particular coordinate not to vary by identifying this coordinate as the one that corresponds to negative curvature on the energy surface at the true saddle point. 